\title{
Mercuryplane-A Spacecraft for Regular Delivery of Astronauts onto the Mercury
}

\author{
Alexander Rubinraut \\ Design Office, Expeditions to Planets (EXPLANET), Munich, Germany \\ Email: rubinraut32@mail.ru
}

How to cite this paper: Rubinraut, A. (2020) Mercuryplane-A Spacecraft for Regular Delivery of Astronauts onto the Mercury. Advances in Aerospace Science and Technology, 5, 71-84.

https://doi.org/10.4236/aast.2020.53005

Received: June 12, 2020

Accepted: July 13, 2020

Published: July 16, 2020

Copyright $\odot 2020$ by author(s) and Scientific Research Publishing Inc. This work is licensed under the Creative Commons Attribution International License (CC BY 4.0).

http://creativecommons.org/licenses/by/4.0/

(c) (i) Open Access

\begin{abstract}
The project of "Mercuryplane" - a spacecraft for regular delivery of astronauts to the planet Mercury is considered. In the first stage of the flight, with the help of a carrier rocket, equipped with a chemical rocket engine, astronauts are delivered to the international space station ISS. A design consisting of an interorbital module and a takeoff-landing capsule has been developed to deliver astronauts from Earth's orbit into Mercury's orbit. The interorbital module is an electric rocket equipped with 8 superconductor magnetoplasma electric engines MARS type. The electric power supply for the engines is provided by a collapsible solar panel made of gallium arsenide. The design of the takeoff-landing capsule has been developed for landing and take-off from the surface of Mercury. A device has been developed to refuel in Earth's orbit the electric rocket engines with nitrogen, and chemical rocket engines with oxygen and hydrogen, which are in liquid state The developed spacecraft is able to regularly deliver three astronauts from Earth orbit to the surface of Mercury within 35 days.
\end{abstract}

\section{Keywords}

Mercuryplane, Superconducting Magnetoplasma Electrorocket Engine MARS, Interorbital MODULE, Takeoff-Landing Capsule,

Solar Battery Made of Gallium Arsenide

\section{Introduction}

The paper is devoted to the problem of solar system mastering by means of sequential carrying out the expeditions towards planets, satellites and large asteroids. This way of actions was outlined as far back as at the twentieth century end when have appeared numerous projects of flights towards Mars planet [1].

But unfortunately after visiting in year 1986 of the only space object-the Moon 
the astronautic has practically stopped in its development. The space firms in different countries had made choice in favour of usage of robots instead of human beings for carrying out planets researches. It was stimulated by the rapid progress in robotics technic development at the twentieth century end. At the same time medical researches, which were carried out at the orbital international space station, have shown that durational (during a year) stay in weightlessness is dangerous for human being health. This fact leads to necessity of the spacecrafts creation which can provide the sufficient conditions for comfort and safety of astronauts during durational flights in the solar system.

The first condition from this point of view is the flight implementation during a minimal time interval. The second necessary condition is that the cabin in which the astronauts are located shall have a system of artificial gravitation as well as a system of protection against dangerous space radiation.

At present the most important factor, which restrains astronautics development, is too high price of spacecrafts launching. In addition now it is absolutely clear that NASA designers and developer have chosen the way of heavy spacecrafts creation with payload more than 100 tons. For launching in space such heavy apparatus one needs huge carrier rockets which creation cost exceeds all the reasonable possibilities and limits.

At the same time assemblage of the space apparatuses by means of their docking on circumterrestrial orbit is carried out already more than 50 years. Thus there is no necessity to spend means on new carrier rockets creation. One can use the carrier rockets which are already in operation and are capable to delivery on circumterrestrial orbit the payload up to 60 tons. In his developments of space apparatuses the author of this paper has chosen exactly this path.

The first step on this path was invention, development and study of the superconducting rocket electro engine of large power named "MARS".

Due to new design of its magnetic system made of superconductor the electrorocket engine of magnetoplasma type was created, which made it possible flight from Earth orbit to orbit almost of any planet of solar system [2].

The second step was projecting and development of the universal interorbital rocket ER7. As engines at the rocket ER-7 the superconducting rocket electro engine "MARS" are installed and as electrical power source the semiconductor solar battery made of gallium arsenide is used.

The conducted researches have shown that on rocket ER7 basis it is possible by means of assemblage of individual units to form space apparatuses of the different assignation. As a result of such designing during the recent years the spacecrafts were developed intended for flights from the Earth orbit to the Moon orbit [3] and from the Earth orbit to the Mars orbit [4].

Now the time had come to develop on basis of conducted researches the spacecraft for flight from the Earth orbit to the Mercury planet orbit.

Creation of spacecraft design for carrying out regular flights onto planet Mercury is the object of this paper. 


\section{The Concept of Astronauts Delivery onto Planet Mercury}

For astronauts delivery onto planet Mercury surface in the project the spacecraft "Mercuryplane", which general view is shown in Figure 1 is developed.

The "Mercuryplane" is an electric rocket, consisting of an interorbital module 1 and a takeoff-landing capsule (TLC) 9. For rocket movement fulfilment the interorbital module 1 has electric rocket engines 5, The electric power supply of the rocket engines is provided by a solar battery 2 made of arsenide gallium.

The working substance (nitrogen in liquid state) for electrorocket engines operation is in a cryogenic tank 13, which is located along longitudinal axis of the rocket. The astronauts delivery onto Mercury surface is performed with the help of the takeoff-landing capsule 9 , which is connected with the rocket 1 by means with docking unit 6 .

During the flight the astronauts are located in a cabin inside of the capsule 9. In the cabin there are control desk as well the on-board life support system. The artificial gravitation inside the cabin during the flight is provided by means of rotation of takeoff-landing capsule in superconducting bearing, which is installed along longitudinal axis.

For landing onto Mercury surface and taking off from its surface the takeoff-landing capsule has hydrogen-oxygen chemical rocket engine 10.

The calculations had shown that the flight onto Mercury is reasonably to perform by stages. At the first stage of the flight the rocket 1 is delivered on the Earth orbit being located (in assembled form) in head part of carrier rocket Delta IV Heavy. At the second stage of the flight the group of astronauts of 3 person is delivered onto the international space station ISS, which is located on Earth orbit. The composition of the group: pilot astronaut, navigator-astronaut and astronaut-researcher. Delivery of the astronauts into orbit is performed in the capsule "Orion" by means of the carrier rocket "Atlas V". At the third stage of the flight with the help of the carrier rocket "Delta IV Heavy" the takeoff-landing 9 is put into the ISS orbit. Further assemblage of the Mercuryplane is made on ISS orbit, At first at the electric rocket 1 the unfolding of the solar battery 2 is being carried out making use the telescopic system 3.

Then the takeoff-landing capsule 9 with the help of chemical rocket engine 10 approaches to an ISS moorage. With the help of the nasal docking unit 11 of the takeoff-landing capsule 9 the docking of the takeoff-landing capsule with the ISS takes place.

Then astronauts pass from the ISS into the takeoff-landing capsule and take their places in crew cabin. The pilot-astronaut takes his place by Mercuryplane control panel. He switches on the chemical rocket engine 10 and the takeoff-landing capsule departs the ISS moorage and is going to the rocket 1 . The pilot-astronaut while being in cabin of the takeoff-landing capsule remotely switches on electric rocket engines 5 of rocket 1 . He is carrying out approach of the rocket 1 with the takeoff-landing capsule (TLC) and then the rocket connection with the TLC with the help of docking unit 12. Thus the assembling of the Mercuryplane on the ISS orbit is being completed. 


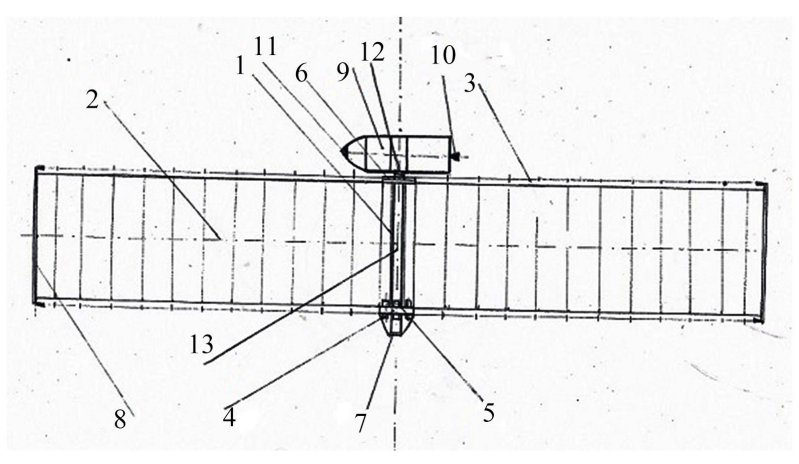

Figure 1. General view of the Mercuryplane.

After switching on of artificial gravitation system in Mercuryplane cabin the TLC begins to being rotated relative to the rocket longitudinal axis with rotational frequency $10 \mathrm{rev} / \mathrm{min}$.

The fourth stage of the flight begins. The pilot-astronaut switches on electric rocket engines 5 and the Mercuryplane begins the movement from Earth orbit towards Mercury orbit.

Now it let us remember the Mercury astronomical data. Mercury planet is the nearest one to the Sun. The velocity of Mercury movement around the Sun is 48 $\mathrm{km} / \mathrm{sec}$. The Mercury, which diameter is $4886 \mathrm{~km}$, is flying around the Sun along an elongated elliptical orbit being approaching to the Sun up to the distance 0.3 A.U. and being moved away from the Sun up to the distance 0.46 A.U. One revolution around the Sun takes 88 earth days. The first research of the Mercury was performed by NASA in year 2011 with the help of the spacecraft "Messenger". It was found that the Mercury rotates around its axis making one turn in 176 days. The inclination of the Mercury orbit to the plane of the ecliptic is only $7^{\circ}$. Because of this reason at the Mercury there are no year seasons like at the Earth. The researches have also shown that the Mercury has practically no atmosphere. At the daytime the temperature at its surface reaches $340^{\circ} \mathrm{C}$. At the night the Mercury surface is cooling up to $-170^{\circ} \mathrm{C}$.

The Mercury surface resembles surface of the Moon, but at the Mercury there are mountain formations having the length in hundreds $\mathrm{km}$ and the height up to $3 \mathrm{~km}$. The acceleration of gravity at the Mercury surface is the same as at Mars and is equal to $3.8 \mathrm{~km} / \mathrm{sec}$.

This short review of the nature conditions at the Mercury clearly shows that the stay on the Mercury is possible only during the night time when sunny rays not achieve his surface. Therefore the mercuryplane landing should be carried out onto the part which is not sunlit.

The duration of the night at the Mercury is equal to 88 earth days. The calculation of the trajectory flight from Earth orbit onto Mercury orbit is carried out with an astrodynamical program, which determinates the picture of gravitation field and the interaction forces of the moving mercuryplane with the Sun, as well with planets Mercury, Venus and Earth at their continuous movement along orbits [5]. 
For calculation the flight trajectory a special program had been developed, which takes in account the continuous change of the mercuryplane mass at operation of electrorocket engines as well the tractive force change of the electrorocket engines at every trajectory point in dependence from distance to the Sun. The significant change of the tractive force mercuryplane is connected with operation of the solar battery, which is energy source of the electrorocket engines being installed at the mercuryplane. It should be remembered that solar energy flow at the Mercury orbit is increased by seven times as compared with the solar energy flow on the Earth orbit which is equal to $1.35 \mathrm{kw} / \mathrm{m}^{2}$. The calculations has shown that for optimal usage of solar energy during the flight it is necessary to increase in twice the number of electrorocket engines 5 , which are installed on the front end disk of rocket (Figure 1).

At the process of designing the calculation of optimal flight trajectory of the mercuryplane was performed, which is provided by means of gradual engines switching on (beginning from 2 and up to 8). At Figure 2 one can see the movement trajectory of the planets Earth, Venus and Mercury around the Sun as well the built trajectory of the Mercuryplane.

At Figure 3 it is shown the change of the movement speed (V), the electrorocket engines power $(\mathrm{P})$ and the Mercuryplane mass $(\mathrm{G})$ during the flight.

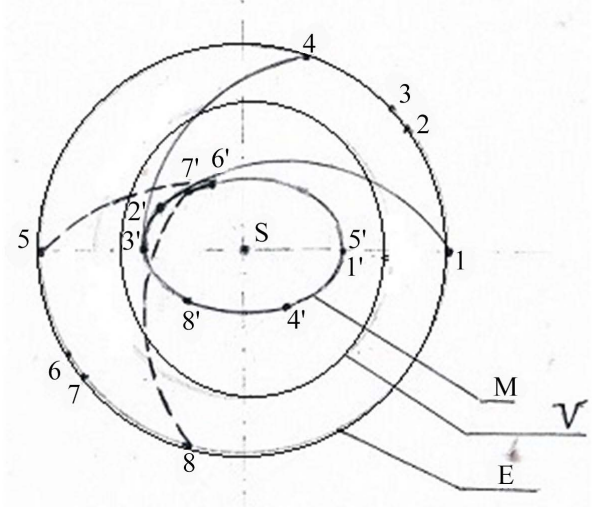

Figure 2. Movement trajectories of planets and the Mercuryplane.

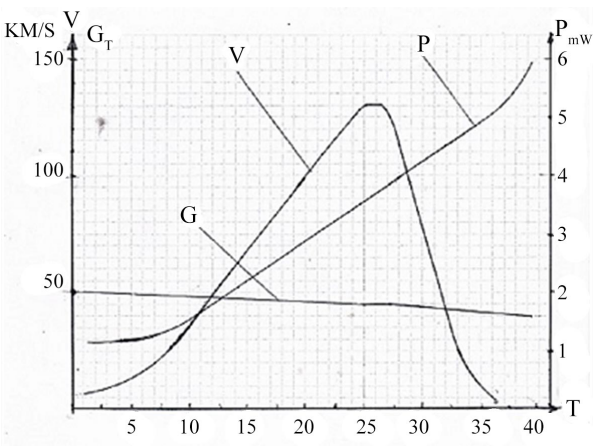

Figure 3. Changing of the Mercuryplane parameters during flight (Earth orbit-Mercury orbit). 
Beginning of the Mercuryplane interorbital flight is planned at the moment of the planets opposition, when the Earth is in the point 1 and the Mercury is in the point 1' (Figure 2). Under action of the electrorocket engines the Mercuryplane begins to increase its speed. The speed at the point 1 exceeds the second space speed at the Earth orbit, the power of electrorocket engines is $1.5 \mathrm{MW}$ and the mass is $50 \mathrm{t}$. The acceleration of the Mercuryplane lasts 25 days. During this time period the power of the electrorocket engines reaches 3.6 MW and movement speed reaches $130 \mathrm{~km} / \mathrm{sec}$. The Mercuryplane crosses orbit of Venus and moves towards the point 2' on the Mercury orbit. In 25 days after flight beginning the electrorocket engines 5 (Figure 1) are switched off. During two days the Mercuryplane continues to fly by inertia with constant speed $130 \mathrm{~km} / \mathrm{sec}$. In 28 days after flight beginning the electrorocket engines are switched on. The Mercuryplane makes 180 degree turn. The power of electromotors at this moment is 4.0 MW. All the 8 electromotors installed on the rocket are operating. The rocket begins to move in brake mode.

In 35 days after flight beginning the speed of the Mercuryplane decreases up to $3.1 \mathrm{~km} / \mathrm{sec}$ while the power of electromotors reaches maximal value- $5 \mathrm{MW}$. The Mercuryplane approaches towards planet Mercury and goes into its orbit in the point $2^{\prime}$. At this moment the Earth while moving along its orbit around the Sun is in the point 2 .

The Mercuryplane while moving along orbit around Mercury at the distance $50 \mathrm{~km}$ from its surface makes one turn in 88 minutes. The pilot-astronaut switches off the system of artificial gravitation and the takeoff-landing capsule stops rotation around longitudinal axis of the Mercuryplane. The separation of the Mercuryplane takes place with the help of docking unit 3 (Figure 1): After the separation the electrorocket engine 5 is switched on.

The speed of rocket ER-7 is increased and it moves off from the takeofflanding capsule 9. Chemical rocket engine 10 of the takeoff-landing capsule is being switched on. The movement speed of the takeoff-landing capsule is decreased and when the speed reaches $3 \mathrm{~km} / \mathrm{sec}$ the takeoff-landing capsule losts weightlessness.

With the help of chemical rocket engine 10 the capsule 9 smoothly moves towards Mercury surface and simultaneously its horizontal movement takes place. The pilot and the navigator are leading the capsule 9 towards a point on the dark side of the Mercury which is located in the internal region of vast crater "Tolstoy" having the coordinates $165^{\circ} /-15^{\circ}$ on Mercury surface [6]. The ER-7 rocket remains in a circular orbit around Mercury. The landing on the surface of Mercury occurs 35 days after the start of the Mercuryplane from the Earth's orbit.

Astronauts in soft heated spacesuits go into the surface of Mercury, which is illuminated by spotlights installed on the side surface of the takeoff-landing capsule. The lighting devices are powered by batteries installed on board the TLC.

Astronauts begin to perform the program of stay on Mercury, which is designed for 7 days. The program is compiled by the company that organizes space flight. It can be both a scientific expedition and a tourist trip. After completion 
of the flight program, the astronauts take their seats in the cabin of the takeoff-landing capsule. After issuing the command "takeoff", the chemical rocket engine 10 (Figure 1) is switched on. The takeoff-landing capsule 9 takes off from the surface of Mercury and enters its orbit, along which is moving the electric rocket ER-7.

After approaching by maneuvering with the help of chemical rocket engine 10, the capsule 9 is being connected with an ER-7 rocket using the docking unit 6, as shown in Figure 1.

The Mercuryplane is now in its assembled form. There are 7 tons of liquid nitrogen left in the cryogenic tank-the working substance for the return flight. The mass of the Mercuryplane has been decreased up to 35 tons.

The system of artificial gravity is switched on and the new stage-flight from Mercury's orbit to Earth's orbit-begins. Let us follow this process of flight of the Mercuryplane in the space and in time, with the help of Figure 2 and Figure 4.

its trajectory around the Sun from point 2' to point 3'. At this time, the Earth is on its trajectory at point 3 . Now the trajectory of the Mercuryplane connects point 3 ' with point 4 in the Earth's orbit around the Sun.

As it can be seen from Figure 4, the first part of the flight towards the Earth takes place within 9 days. The Mercuryplane with the help of electric rocket engines 5 is accelerated up to the speed of $125 \mathrm{~km} / \mathrm{s}$.

For maximum use of the Sun's energy, 8 electric rocket engines are turned on at full power. At the beginning of the flight, the solar panel has a $6 \mathrm{MW}$ capacity. After 9 days of flight, the power is being reduced to $4 \mathrm{MW}$. At this time, the Mercuryplane reaches the orbit of Venus.

Electric rocket engines are switched off, and within 2 days the Mercuryplane flies by inertia at speed of $125 \mathrm{~km} / \mathrm{s}$. In 2 days the prolonged braking part of the flight begins. The Mercuryplane makes a 180-degree turn. The electric rocket engines 5 are being switched on and the Mercuryplane reduces speed by moving towards the point 4 in Earth orbit (Figure 2).

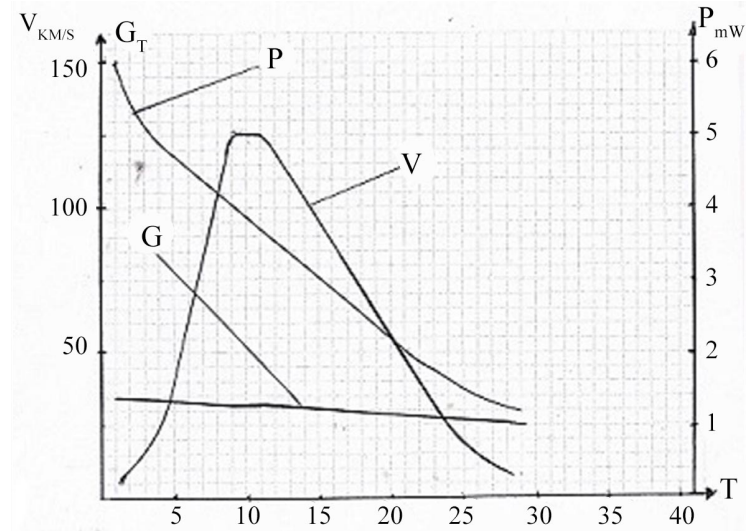

Figure 4. Changing the parameters of the Mercuryplane (movement speed $(\mathrm{V})$, the electrorocket engines power (P) and the Mercuryplane mass (G)) in the return flight (Mercury orbit-Earth orbit). 
The last section of the trajectory of the Mercuryplane passes in 17 days. During this period the power of electric motors is reduced to 1.5 MW. On the 29th day of the flight, the Mercuryplane reaches the Earth and enters the orbit of the ISS located at a distance of $370 \mathrm{~km}$ from the Earth's surface. The takeoff-landing capsule, which during all the flight was rotating around the longitudinal axis, creating artificial gravity in the astronauts' cabin, stops. With the help of the docking unit 12 the capsule is being disconnected from the electric rocket 1. After turning on the electric rocket engines 5 the rocket 1 departs from the takeoff-landing capsule. With the help of a chemical rocket engine 10 TLC moves to a moorage of the ISS and connects with it. The astronauts pass from the TLC inside the ISS. On another ISS moorage the astronauts which have arrived from Mercury are already waited by takeoff-landing capsule "Orion", which delivers them to the surface of the Pacific Ocean.

The developed project of the Mercuryplane provides regular flights of astronauts onto Mercury. The research calculations have shown that it is reasonable to fly to Mercury twice a year. In order to make certain in it, let's refer to Figure 2, where a solid line shows the trajectory of the first, and the dotted line of the trajectory of the re-flight to Mercury. The first flight ends with the arrival of the Mercuryplane with astronauts into the point 4 of Earth orbit.

The total time that was spent for the flight and the astronauts' stay on the surface of Mercury was 72 days. During this time, the planet Mercury while moving along its orbit around the Sun had traveled from point 1' to point 4'.

Three months after the first flight the favorable location of the planets for the second flight from the Earth's orbit arises again. The second flight from Earth's orbit should be begun, when the Earth is at point 5, and Mercury is in its orbit at point 5 .

The calculations showed that the flight time along the second trajectory from point 5 in Earth orbit to point 6 ' in Mercury orbit will take 32 days.

The landing of the second group of astronauts on the surface of Mercury is expected to carry out at point 6 .

After 7 days of staying on the surface of Mercury, the Mercuryplane again takes course for Earth orbit. The interorbital flight is carried out on along the calculated trajectory from point 7 ' to point 8 in Earth's orbit and takes 37 days. Thus, the full staying time of the second group of astronauts in space flight will be 76 days.

Figure 2 shows that at the end of the second flight Mercury will be located in its orbit at point 8 '.

The next third regular flight of the Mercuryplane is advisable to start in 3 months from point 1 in Earth orbit. As a result of calculated research, in the project a system of regular flights to the surface of Mercury was developed.

After the first orbital flight, the Mercuryplane is in orbit around the Earth. The tanks with a working substance-nitrogen for electric rocket engines, as well as the tanks located in the TLC with hydrogen and oxygen for the operation of chemical rocket engines are empty. 
In order to implement re-fly the Mercuryplane into the orbit of the ISS, a space refueler filled with nitrogen, oxygen and hydrogen, which are in liquid condition, is delivered.

The scheme of refueling the Mercuryplane by the working substance for the operation of the engines is shown on Figure 5.

The refueling of the Mercuryplane is controlled by the pilot-astronaut, which is located in TLC 4 . With the help of docking unit 3 he docks the TLC 4 with the space refueler 2 .

Next, the TLC 4 with the refueler 2 departs from the moorage TLC, and with the help of a chemical rocket engine 8 is headed to the electric rocket 1 and docks with it, as shown in Figure 5. After docking with the help of the docking unit 3, the connection of the tank with liquid nitrogen of the refueler with flexible cryogenic pipeline located on the longitudinal axis of the electric rocket 1 takes place. In order to connect the nitrogen cryogenic pipelines in the project a special device has been developed. With the help of remote control, the astronaut-pilot switches on the cryogenic pump located in a nitrogen pipeline. The liquid nitrogen is being pumped from the tank of the refueler into the cryogenic tank of electric rocket 1 . The design of the refueler 2 is given below.

After refueling the electric rocket with nitrogen, TLC 4 with the refueler 2 depart from electric rocket 1 .

The pilot-astronaut, who is in cabin of the TLC, connects flexible cryogenic hoses passing through the docking unit 9, through which liquid hydrogen and liquid oxygen are entering into the tanks located inside the TLC hull. The design of the TLC is given below.

After refueling of the chemical rocket engine 8 by fuel and oxidizer the space refueler 2, along with TLC 4, moves towards the ISS. Eventually the refueler 2 and TLC 4 are beiblocated on different moorages of the ISS.

After mooring the refueled takeoff-landing capsule of the Mercuryplane is in readiness to send a new team of astronauts to Mercury. The electric rocket of the Mercuryplane is also ready to fly into Mercury's orbit. It is in orbit of the ISS moving around the Earth at a distance of $1 \mathrm{~km}$ from the ISS.

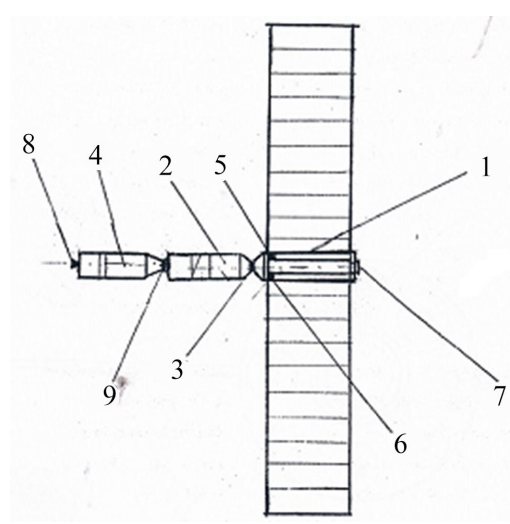

Figure 5. The scheme of refueling the Mercuryplane by working substance. 
As it can be seen from Figure 2, the third regular flight to Mercury is expected on the trajectory of the first flight from point 1 to point 2'. Thus, the possibility of conducting multiple regular flights from the Earth's orbit to the surface of Mercury and back is realized.

Now let's try to calculate the cost of regular flights of the Mercuryplane. The flight onto Mercury will require two launches of the Delta IV Heavy carrier rocket, which will cost 300 million dollars.

The delivery of astronauts to the ISS and back to the Earth surface with the Atlas V rocket will cost 100 million dollars. The calculated cost of the ER-7 rocket with 8 engines is 250 million dollars. The calculated cost of the refueler is 100 million dollars. The cost of the takeoff-landing capsule is 80 million dollars. The projective cost of one flight of the Mercuryplane will be 850 million dollars.

Thus, it should be expected that the use of a new space device-the Mercuryplane will speed up the process of human mastering of the solar system. Implementation of this project will allows to regularly visit Mercury-the planet of the solar system, which is closest to the Sun.

\section{The Structure of the Mercuryplane}

The general view of the Mercuryplane is shown at Figure 1 (see page 74).

1) Interorbital module

2) Solar panel made of arsenide gallium

3) Telescopic system of the solar panel unfolding

4) Front end disk

5) Electrorocket engine

6) Rear end disk

7) Nasal docking unit

8) Support beam of solar panel

9) Takeoff-landing capsule

10) Chemical rocket engine of takeoff-landing capsule

11) Nasal docking unit of takeoff-landing capsule

12) Side docking unit of takeoff-landing capsule

13) Cryogenic tank with working substance-liquid nitrogen

The design solution of the Mercuryplane is based on the usage of the developed by the author rocket, ER-7, the detailed description of which was first published in [7].

This paper shows the differences and new design decisions that have been made to meet the requirements of the conditions on Mercury.

Figure 6 shows the location of sliding rods of the solar panel and electric rocket engines in the cross-section of the electric rocket.

The Mercuryplane is equipped with 8 electric rocket engines of the type "MARS", the design of which was developed by the author in 2006-2013. MARS type engines belong to the class of superconductor magnetoplasma electrorocket engines. 
Detailed description of the design of the "MARS" electric engine is available in the [2] and [7] and its main parameters are given in Table 1.

The main characteristics of the interorbital module of the Mercuryplane 1 (Figure 1) are given in Table 2.

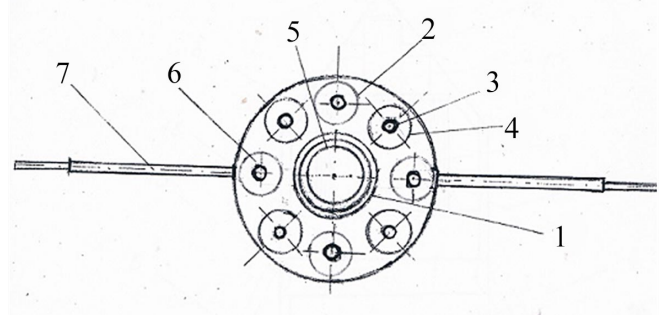

Figure 6. Location of sliding rods of solar panel and electric rocket engines. 1: Cylindrical hull of rocket; 2: Superconductor electric rocket engine; 3: Jet nozzle of electric rocket engine; 4 : The outer shell of the electric rocket engine; 5: The inner shell of a cryogenic tank with liquid nitrogen; 6: The outer shell of the cryostat, inside which is installed superconducting exciting coil of the electric rocket engine; 7: Telescopic rod for solar panel unfolding.

Table 1. Parameters of the electric engine "MARS".

\begin{tabular}{cc}
\hline Tractive force & $80 \mathrm{~N}$ \\
\hline Power & $800 \mathrm{~kW}$ \\
Current & $800 \mathrm{~A}$ \\
Voltage & $1000 \mathrm{~V}$ \\
Efficiency & $95 \%$ \\
Specific impulse & $6600 \mathrm{sec}$ \\
Working substance consumption & $1.2 \mathrm{G} / \mathrm{sec}$ \\
Working substance ejection velocity & $10 \mathrm{~km} / \mathrm{s}$ \\
Magnetic induction & $1.5 \mathrm{~T}$ \\
Cathode diameter & $40 \mathrm{~mm}$ \\
Anode diameter & $180 \mathrm{~mm}$ \\
Anode length & $200 \mathrm{~mm}$ \\
Cathode length & $160 \mathrm{~mm}$ \\
Diameter of external cylinder & $850 \mathrm{~mm}$ \\
Length of external cylinder & $550 \mathrm{~mm}$ \\
\hline
\end{tabular}

Table 2. Characteristics of the interorbital module.

\begin{tabular}{cc}
\hline External diameter & $6 \mathrm{~m}$ \\
\hline Length & $22 \mathrm{~m}$ \\
Solar battery width & $20 \mathrm{~m}$ \\
Solar battery wing length & $60 \mathrm{~m}$ \\
Mass of the interorbital module & $23 \mathrm{ton}$ \\
\hline
\end{tabular}




\section{Takeoff-Landing Capsule of the Mercuryplane}

It is designed to move three astronauts from Earth orbit to Mercury's orbit, as well as for landing and taking off from the surface of Mercury. The design of the capsule is a further modification of the design, which was developed by the author for landing and taking off from the surface of Mars space capsule of marsoplane [4].

The main difference is that the Mercuryplane capsule has a docking unit 16 on load-bearing ring of the landing tripod, which allows to realize the rotation of the takeoff-landing capsule around the transverse axis and create artificial gravity in the astronauts' cabin.

The design of the Mercuryplane's takeoff-landing capsule is shown on Figure 7.

\section{Mercuryplane Refueler Structure}

The refueler is designed to refuel electrical and chemical rocket engines with working substance that is carried out in orbit of the International Space Station according to a diagram shown on Figure 5.

Such refueling is necessary for the re-flight to Mercury. The design of the refueler, made in the form of a system of concentric cryogenic tanks.

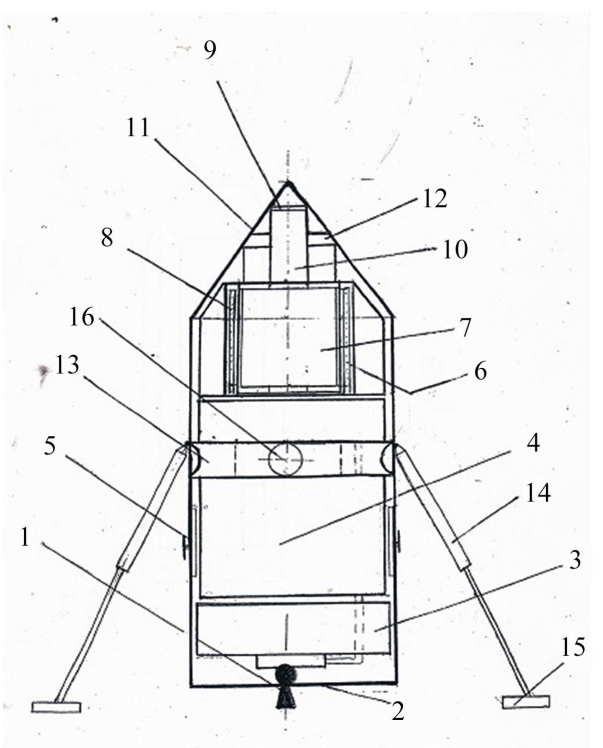

Figure 7. The takeoff-landing capsule of Mercuryplane. 1: Chemical hydrogen-oxygen engine having tractive force $200 \mathrm{KN}$ and specific impulse $400 \mathrm{sec}$; 2: Bottom of the capsula rocket hull; 3: Fuel tank with liquid oxygen; 4: Fuel tank with liquid hydrogen; 5: Chemical rocket engine for maneuver; 6: Tank with liquid hydrogen; 7: Expedition crew cabin; 8: Superconducting solenoid; 9: Docking unit for connection with ISS moorage; 10: Tunnel and gateway for astronauts' exit; 11: The external shell of the hull; 12: Container for measuring devices; 13: Load-bearing ring of landing tripod; 14: Damper of landing rack; 15: Shoe of landing rack; 16: Docking unit for capsule connection with orbital module. The mass of the takeoff-landing capsule on Earth-26 T; The length of the takeoff-landing capsule-17 m; External diameter of the capsule-5.8 $\mathrm{m}$. 


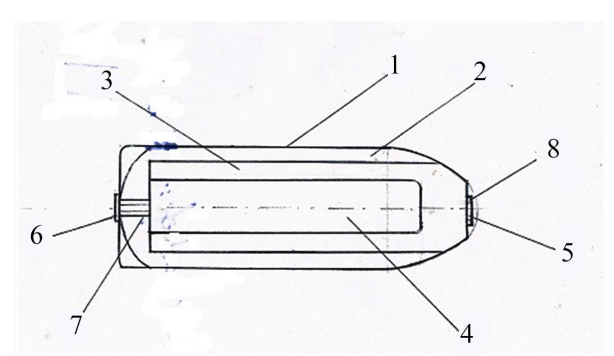

Figure 8. Refueler for Mercuryplane. 1: Outer cylindrical shell; 2: Cryogenic tank with liquid oxygen; 3: Cryogenic tank with liquid nitrogen; 4: Cryogenic tank with liquid hydrogen; 5:Docking unit for connection with orbital module; 6: Docking unit for connection with takeoff-landing capsule; 7: Liquid hydrogen pipeline; 8: Liquid nitrogen pipeline.

The external cryogenic tank is designed to store liquid oxygen. In the middle of the structure is cryogenic tank with liquid nitrogen. Along axis of the refueler the largest tank with liquid hydrogen is located. Cryogenic tanks are made of carbon and have axial cryogenic pipelines. The construction of the refueler is shown in Figure 8.

\section{Conclusions}

A complex of works on the creation of the spacecraft-Mercuryplane for the regular delivery of astronauts to the planet Mercury has been carried out.

The design and basic characteristics of the Mercuryplane are defined, for which:

1) A method of two-stage delivery of astronauts to the surface of Mercury using the ISS as space base has been developed.

2) Comprehensive scientific research and design of the Mercuryplane-a spacecraft for the flight of astronauts from an ISS moorage to the surface of Mercury and back with the help of an electric rocket has been carried out.

3) The calculated studies showed the possibility of conducting two regular flights of the Mercuryplane with landing on the surface of the planet within one year.

4) To land on the surface of Mercury and take off from its surface, the design of a takeoff-landing capsule with an artificial gravity system and with protection of astronauts from cosmic radiation has been developed.

5) The developed design of the Mercuryplane will allow the regular delivery of three astronauts to the surface of Mercury and back to Earth orbit in 65 days.

\section{Conflicts of Interest}

The author declares no conflicts of interest regarding the publication of this paper.

\section{References}

[1] Filser, H. (2009) Die längste Reisen aller Zeiten. GEO Kompakt N21 S98-107, November 2009. 
[2] Rubinraut, A. (2017) The Study of the Electrorocket Engine of the Future. Advances in Aerospace Science and Technology, 2, 1-16. https://doi.org/10.4236/aast.2017.21001

[3] Rubinraut, A. (2019) The Moonplane-A Spacecraft for Regular Delivery Astronauts onto the Moon. Advances in Aerospace Science and Technology, 4, 43-56. https://doi.org/10.4236/aast.2019.43004

[4] Rubinraut, A. (2020) Expedition to Mars North Pole and Creation: There Is a Scientific Research Base. Advances in Aerospace Science and Technology, 5, 20-43. https://doi.org/10.4236/aast.2020.51002

[5] Рой (1991) Движение по орбитам Мир. Москва. (In Russian)

[6] Krisciunas, K. and Yenne, B. (1998) Atlas des Universums, Lechner. 5-18.

[7] Rubinraut, A. (2018) The Space Cruise over the Route "Earth-Mars-Earth". Advances in Aerospace Science and Technology, 3, 20-47.

https://doi.org/10.4236/aast.2018.32003 